\title{
Estimación de la capacidad antioxidante de los alimentos ingeridos por la poblaclión española
}

\section{Estimation of the antioxidant capacity of foods consumed by the spanish population}

\begin{abstract}
In the body, the reactions by which energy is created produce reactive oxygen molecules (ROS). ROS can damage biomolecules such as lipids, proteins, carbohydrates, proteins and DNA, which has been linked to chronic diseases such as cancer, cardiovascular diseases, inflammatory processes, diabetes and aging. Antioxidants present in foods can counteract the harmful effect of ROS. The aim of this study was to quantify the average intake of antioxidant capacity in the Spanish diet. For this purpose, average daily food intake published in the National Survey of Spanish Dietetics in 2011 and the database that has been published by the USDA (based on the ORAC) was used. The Spanish population surpassed the estimated daily recommendation of antioxidants and was superior to other countries that have similar diets.
\end{abstract}

Key words: nutrition; antioxidant capacity; ORAC; adults; diets

\section{INTRODUCCIÓN}

Las sociedades actuales se enfrentan a un problema mundial como son las enfermedades crónicas tales como la diabetes, el cáncer y las enfermedades cardiovasculares'. Una de las teorías más aceptada es que el estrés oxidativo está involucrado en la etiología de las enfermedades crónicas $^{2-3}$.

Los antioxidantes pueden contrarrestar los efectos del estrés oxidativo bien por inhibición o por evitar la propagación de las especies reactivas de oxígeno, previniendo y/o reparando los daños que estas moléculas producen ${ }^{4}$. Consistentes estudios epidemiológicos han sugerido que las moléculas antioxidantes presentes en los alimentos, pueden prevenir el daño oxidativo derivados de estas reacciones y por tanto tienen un efecto preventivo frente a las enfermedades crónicas ${ }^{5-6}$. Este efecto protector no solo es debido a sus nutrientes (vitaminas, minerales, péptidos, etc) sino también a otras moléculas denominadas fitoquímicos entre los que se incluyen los compuestos fenólicos y los carotenoides ${ }^{7}$. Además de las frutas, verduras
Inmaculada Navarro-González, María Jesús Periago, Francisco Javier García-Alonso

\footnotetext{
Departamento de Tecnología de los Alimentos, Nutrición y Bromatología. Área de Nutrición y Bromatología. Facultad de Veterinaria. Universidad de Murcia. Campus de Espinardo 30100; Murcia, España
}

Dirigir correspondencia a: Inmaculada Navarro-González, Departamento de Tecnología de los Alimentos, Facultad de Veterinaria. Universidad de Murcia. Campus de Espinardo 30100; Murcia, España. Tel: 34868 884798, Fax: 34868888497. Email: inmaculada.navarro@um.es Este trabajo fue recibido 02 de marzo 2017
Aceptado con modificaciones 07 de mayo de 2017,
aceptado para ser publicado 16 de mayo de 2017.

y vegetales, hay otros alimentos que también tienen una elevada capacidad antioxidante como el té, el vino, el café, y los zumos de frutas ${ }^{7-8}$.

Debido a la dificultad de medir por separado la capacidad antioxidante de las moléculas y sus efectos sinérgicos, se han desarrollado distintos métodos para evaluar su capacidad antioxidante total ${ }^{9}$. Uno de los métodos más empleados para medir la capacidad antioxidante en alimentos es el ORAC (Capacidad de Absorción de Radicales Libres) debido a que permite comparar alimentos de distinta naturaleza en cuanto a su riqueza en moléculas antioxidantes, y a que mide la capacidad de los antioxidantes presentes en la muestra para proteger una proteína (fluoresceína) del daño oxidativo. Debido a su reconocimiento en el año 2007, la USDA (Departamento de Agricultura de los Estados Unidos) publicó la primera base de datos de la actividad antioxidante de 277 alimentos seleccionados utilizando la metodología ORAC ${ }^{10}$. Por otro lado, el Laboratorio de Análisis de Antioxidantes del Instituto de Nutrición y 
Tecnología de los Alimentos (INTA), Universidad de Chile, también han creado una base de datos online de actividad antioxidante (ORAC) y del contenido en polifenoles totales en frutas producidas y/o consumidas en Chile, actualizada en el año $2013^{11}$.

El objetivo general de este trabajo ha sido evaluar la capacidad antioxidante ORAC basándonos en la ingesta media de alimentos por día de los españoles publicada en la Encuesta Nacional de Dietética Española (ENIDE) del año 2011.

\section{MATERIALES Y METODOS}

Utilizando los datos de la encuesta ENIDE (Encuesta Nacional de Dietética Española) de España, se evaluó la capacidad antioxidante de los alimentos ingeridos por la población española ${ }^{12}$. Dicha encuesta fue realizada y publicada por la Agencia Española de Seguridad Alimentaria y Nutrición (AESAN), cuyo objetivo es determinar los patrones de ingesta dietética en la población española $(n=3000)$ por grupos de edad (18-24 y $25-65$ ) y sexo (hombre-mujer), obtener datos que ayudan a evaluar el estado nutricional y la ingesta de macro y micronutrientes de dicha población.

Los datos de consumo de alimentos, de la encuesta, se obtuvieron con un recordatorio 24 horas completado por cada participante con la ayuda de un encuestador entrenado, así como el registro de consumo de tres días no consecutivos (incluido uno festivo). La encuesta ENIDE distribuye los datos de ingesta agrupando los alimentos en 12 grupos (bebidas sin incluir leche; huevos y ovoproductos; frutas y productos frutícolas; cereales; carne y productos cárnicos; leche, lácteos y sustitutivos de la leche; miscelánea, referido a productos preparados tales como salsas, ensaladas y postres dulces; frutos secos y semillas oleaginosas; productos del mar o relacionados; azúcar y productos azucarados; y verduras y vegetales). Cada alimento se expresa en g (para los sólidos) o ml (en caso de líquidos) por persona y por día, teniendo en cuenta la población general (consumidores y no consumidores). Los grupos de alimentos seleccionados para evaluar su capacidad antioxidante han sido el de las bebidas sin incluir leche, frutas y productos frutícolas, cereales, miscelánea, frutos secos y semillas oleaginosas, azúcar y productos azucarados, y verduras y vegetales; ya que son los alimentos que presentan mayor cantidad de compuestos bioactivos con actividad antioxidante ${ }^{7-8}$.

La base de datos empleada para poder evaluar la capacidad de antioxidante teórica de los alimentos ingeridos por los españoles fue la publicada por la USDA (la cual utiliza la metodología ORAC).

En este trabajo se calcularon los valores teóricos para ORAC-hidrofílico (H-ORAC), ORAC-lipofílico (L-ORAC), ORAC totales y para algunos alimentos los fenoles totales (TP), en función de los gramos de alimentos consumidos por los españoles según la ENIDE. H-ORAC, L-ORAC y el total de ORAC se expresan en mol de equivalentes de Trolox por cada 100 gramos $\left(\mu \mathrm{mol}\right.$ TE/100 g) ${ }^{10}$. Para cuantificar cuantitativamente el contenido en compuestos fenólicos de algunos alimentos, se empleó la base de datos Phenol-Explorer $3.6^{13}$. Los fenoles totales se expresan en equivalentes de ácido gálico por cada $100 \mathrm{~g}$ (mg GAE/100 g).

\section{RESULTADOS}

En el presente trabajo se estimó la capacidad antioxidante que tiene la dieta de los españoles, distinguiendo el aporte hidrofílico y lipofílico. Tal y como se muestra en la tabla 1, la capacidad antioxidante ORAC de la ingesta media estimada por persona y día fue en torno a los $10.000 \mu \mathrm{mol}$ $\mathrm{TE}$, siendo prácticamente la totalidad de origen hidrofílico $(98 \%)$ frente a un $2 \%$ de origen lipofílico. Los grupos de alimentos que más contribuyeron a la actividad ORAC total fueron las frutas, verduras y bebidas (Figura 1).

Dentro del grupo de las frutas, los principales contribuidores a la ingesta de valor ORAC fueron la manzana (31\%) y naranja (20\%), seguidos de la pera $(11 \%)$ y melocotón (8\%) (figura 2A). La ingesta de naranja de la población española fue inferior a la de manzana, unos 35 g/p/día, y su contribución a la actividad ORAC (17\%) también fue inferior.

Dentro de este grupo, se incluyeron algunas frutas que exhiben alta capacidad antioxidante como las uvas, granadas o cerezas, pero su ingesta diaria estimada resultó tan baja que su contribución al valor ORAC total es minoritaria.

En cuanto al grupo de las verduras y vegetales, los alimentos que más contribuyen a la ingesta ORAC fueron la patata $(27 \%)$ y las lentejas $(15 \%)$, junto con la lechuga $(10 \%)$ y el tomate (9\%) (Figura $2 \mathrm{~B})$.

Dentro de las bebidas, los principales contribuidores al índice de riqueza de antioxidantes ORAC fueron los vinos de mesa $(67 \%)$, el té $(18 \%)$ y los zumos de cítricos $(12 \%)$. La ingesta de té de la población española fue menor que la de vino, (unos $31 \mathrm{ml} / \mathrm{p} / \mathrm{día}$ ), y por eso contribuyó con tan solo un $18 \%$ al índice de riqueza ORAC. En tercer lugar están los zumos de cítricos, que contribuyeron con un $12 \%$ al valor ORAC, valor muy próximo al té.

Con respecto al grupo de azúcar y productos azucarados, los únicos contribuidores al aporte de antioxidantes fueron el chocolate (44\%), el cacao $(27 \%)$, bombones $(26 \%)$ y, en menor medida, el chocolate con leche (3\%) (Figura 2C).

Dentro de los cereales, los mayores contribuidores al índice ORAC fueron los cereales de maíz (37\%) y las palomitas (21\%). Aun así, fue un grupo de alimentos con una ingesta media muy baja en la población española, siendo el maíz en conserva el producto de este grupo que más se ingirió y a la vez el que menos valor ORAC aportó.

En cuanto al grupo de misceláneos, el principal contribuidor a la capacidad antioxidante ORAC fueron las salsas con base de tomate, como el tomate frito y la salsa boloñesa con un $82 \%$ (Figura 2D).

Por último, de todos los frutos secos los que más capacidad antioxidante ORAC aportan fueron las nueces $(57 \%)$, las avellanas $(16 \%)$ y las almendras $(13 \%)$.

Dentro del grupo de los frutos secos se incluyeron algunos de alta capacidad antioxidante, como los pistachos, 


\begin{tabular}{|c|c|c|c|}
\hline & nol TE/p/día) es & s en la pob & ñola. \\
\hline GRUPOS & ORAC-Total & ORAC-L & ORAC-H \\
\hline Bebidas & $1.937,5$ & 0,0 & $1.937,5$ \\
\hline Frutas & $3.572,6$ & 63,6 & $3.509,0$ \\
\hline Cereales y otros granos & 48,5 & 3,4 & 45,1 \\
\hline Misceláneas & 125,6 & 4,0 & $1.21,7$ \\
\hline Productos azucarados & $1.155,4$ & 9,4 & $1.146,0$ \\
\hline Verduras y vegetales & $3.130,0$ & 94,6 & $3.035,4$ \\
\hline Total & $10.241,2$ & 186,2 & $10.055,0$ \\
\hline
\end{tabular}

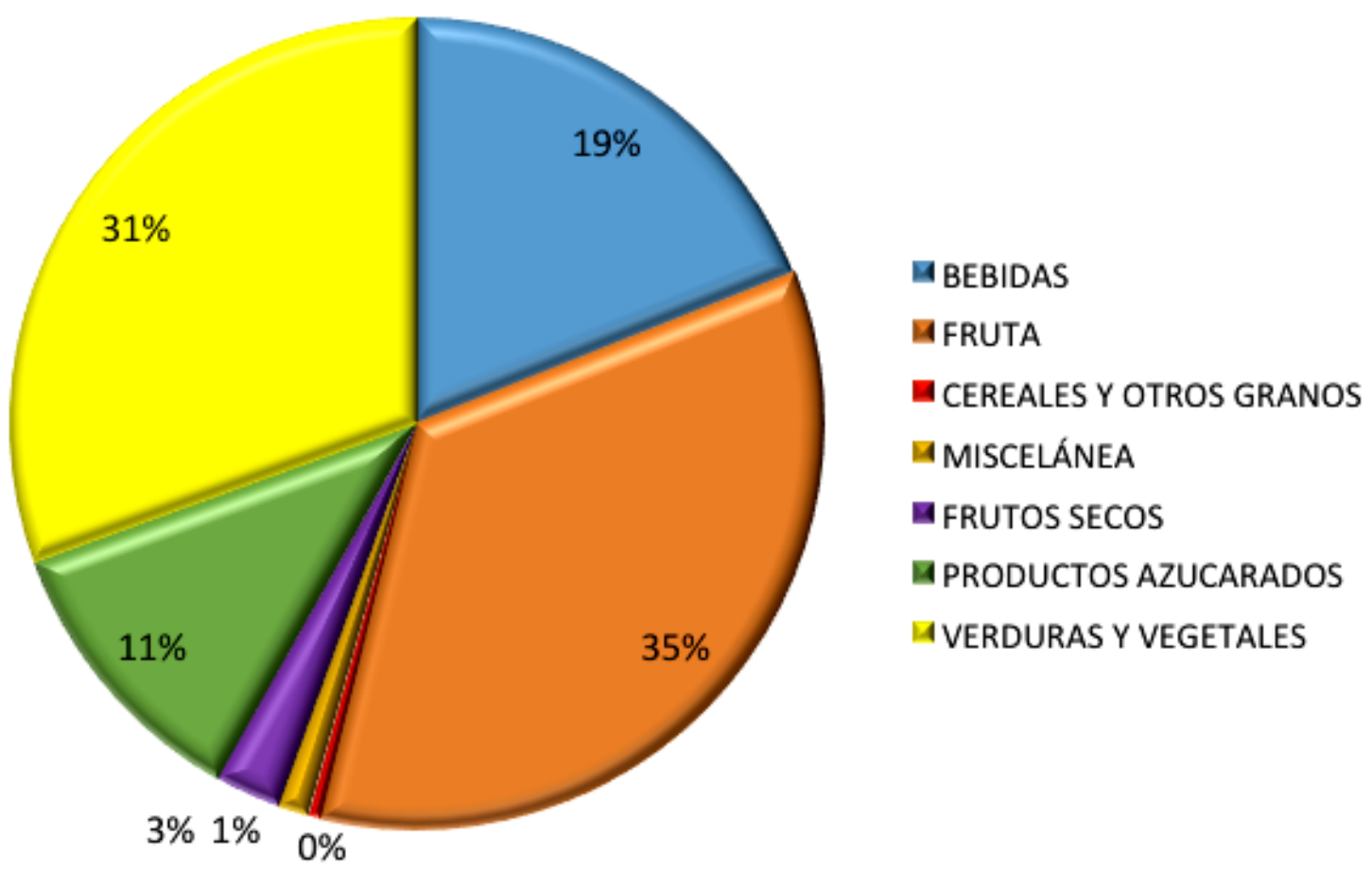

Figura 1. Contribución (\%) de cada grupo de alimentos a la ingesta total ORAC. 


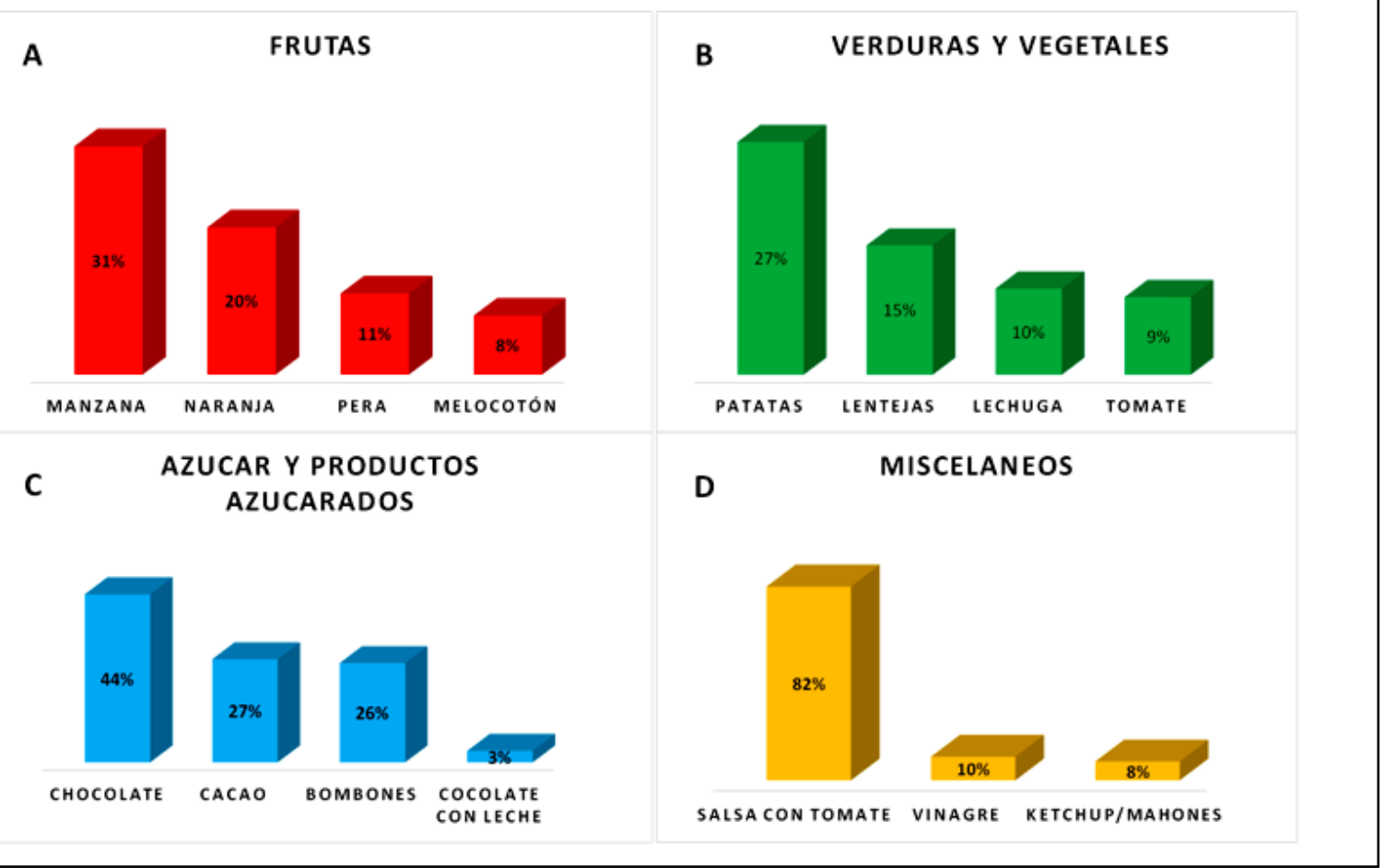

Figura 2. Mayor contribución (\%) de los alimentos ingerido por la dieta dentro de cada grupo de alimentos a la ingesta total ORAC, en los españoles.

cuya cantidad de compuestos fenólicos totales fue superior a la de las nueces, pero su ingesta diaria estimada resultó ser tan baja que su contribución al valor ORAC es minoritaria

\section{DISCUSIÓN}

El valor de la capacidad antioxidante que tiene la dieta de los españoles está en concordancia con los datos estimados para la ingesta media diaria por persona y día de compuestos fenólicos totales $(404 \mathrm{mg} / \mathrm{p} / \mathrm{día})^{13}$ y carotenoides $(4.3 \mathrm{mg} / \mathrm{p} / \mathrm{dí})^{14}$. Tal y como se observa, la ingesta estimada de compuestos fenólicos es del orden de 100 veces superior a la de carotenoides, lo cual podría explicar que el valor ORAC-L estimado sea tan inferior, ya que gran parte de los compuestos fenólicos actúan como antioxidantes hidrofílicos, mientras que los carotenoides actúan como antioxidantes lipofílicos ${ }^{15}$.

Además, esto coincide con las estimaciones de las ingestas españolas de vitamina C (124-139 mg/p/día) y vitamina $E(13-14 \mathrm{mg} / \mathrm{p} / \mathrm{d}$ ía), que son 10 veces superiores en el caso de la vitamina $\mathrm{C}^{16}$, la cual contribuiría de forma importante a los valores de ORAC-H.

Aunque no hay una ingesta dietética de referencia (IDR) para la ingesta de la capacidad antioxidante, si hay algunas recomendaciones basadas en estudios epidemiológicos;
Prior et al. ${ }^{17}$ estimó que debería ingerirse una capacidad antioxidante de $4.6 \mu \mathrm{mol}$ TE por caloría consumida, lo que supondrían unas $9.200 \mu \mathrm{mol}$ TE para una dieta de 2.000 cal. Por ende, podemos afirmar que la población española supera sin ninguna dificultad estas recomendaciones. Sin embargo, diversos estudios similares a éste han demostrado que en países como Grecia, que también siguen una dieta mediterránea, la ingesta diaria de alimentos con capacidad antioxidante es inferior a la española $(6.700 \mu \mathrm{mol}$ TE/ persona/día $)^{18}$.

Por otro lado, muy por debajo de las recomendaciones se encuentra Estados Unidos, donde se ha estimado una ingesta de entre $5.558 \mu \mathrm{mol}$ TE/persona/día ${ }^{19}$.

Dentro del grupo de frutas, la elevada aportación de la manzana al valor ORAC se explicaría por la cantidad diaria ingerida por la población española ( 41 g/p/día) y por su aporte en compuestos fenólicos totales que se encuentra en el rango 210-260 mg/100 $\mathrm{g}^{10}$. La contribución de la naranja a la actividad ORAC (17\%) se explicaría más que por su aporte de compuestos fenólicos ( $57 \mathrm{mg} / 100 \mathrm{~g}$ ), que es muy inferior al de la manzana, por su aporte de vitamina $\mathrm{C}$ que es 7 veces mayor que el de la manzana $(50 \mathrm{mg} / 100$ g vs $3 \mathrm{mg} / 100 \mathrm{~g})^{20}$.

Con respecto al grupo de las verduras, la aportación 
de la patata a la capacidad antioxidante ORAC se puede explicar por la elevada ingesta diaria de la población española ( $66 \mathrm{~g} / \mathrm{p} / \mathrm{día})$, además del aporte de compuestos fenólicos totales encontrado entre el rango 122-179 mg/100 g. Sin embargo, las lentejas que tienen una mayor cantidad de compuestos fenólicos que la patata $(628 \mathrm{mg} / 100 \mathrm{~g})^{10}$, contribuyen menos debido a la menor ingesta por parte de la población española $(7 \mathrm{~g})$. A pesar de que las cantidades ingeridas de tomate, lechuga y cebolla son elevadas, con respecto a otros vegetales, las cantidades consumidas aportan una baja cantidad de antioxidantes ${ }^{21}$.

En el grupo de las bebidas, el vino es la bebida que más valor aporta al índice ORAC, debido a su gran aporte de compuestos fenólicos totales, situado entre 203-228 $\mathrm{mg} / 100 \mathrm{~g}^{10}$, además de ser el alimento que más se ingiere diariamente dentro de este grupo. Aunque los zumos de cítricos contribuyen menos que el vino, su aportación al índice ORAC se debe más que por la cantidad de compuestos fenólicos $(67 \mathrm{mg} / 100 \mathrm{~g})^{10}$, por la cantidad de vitamina C que contienen $(39 \mathrm{mg} / 100 \mathrm{~g})^{19}$.

El índice de riqueza de antioxidante ORAC perteneciente al grupo de azúcar y productos azucarados se debe básicamente a la alta cantidad de compuestos fenólicos totales que contienen, siendo el cacao el que más contiene $(3.663 \mathrm{mg} / 100 \mathrm{~g})^{10}$, aunque la ingesta diaria sea muy baja $(0,6 \mathrm{~g})$. Se trata, con diferencia, del grupo de alimentos que contienen el valor de ORAC más elevado, pero a la vez el grupo de alimentos que menos se ingieren diariamente.

Tanto el maíz como las palomitas (proceden de la misma matriz) contienen una alta cantidad de compuestos fenólicos totales que explicaría su contribución a la capacidad antioxidante ORAC, con una cantidad de $842 \mathrm{mg} / 100 \mathrm{~g}^{10}$.

La contribución de las salsas con base de tomate al índice de riqueza antioxidante ORAC se entendería por la cantidad diaria ingerida por la población (10 g) y por su aporte en compuestos fenólicos totales, unos $245 \mathrm{mg} / 100 \mathrm{~g}$ aproximadamente 10 . Además, la salsa boloñesa es fuente de vitamina $A(215,5 \mu \mathrm{g} / 100 \mathrm{~g})$ y el tomate frito de vitamina $\mathrm{C}(17,5 \mathrm{mg} / 100 \mathrm{~g})^{20}$ y licopeno ${ }^{21}$.

La aportación de las nueces, dentro del grupo de los frutos secos, al valor ORAC se puede justificar por su alta cantidad de compuestos fenólicos totales, que es de unos $1.556 \mathrm{mg} / 100 \mathrm{~g}^{10}$, a pesar de que la ingesta en la población española no es muy elevada (1,2 g/p/día). La ingesta de avellanas es inferior a la de las nueces, unos $0,8 \mathrm{~g} / \mathrm{p} / \mathrm{día}$, y su contribución a la capacidad antioxidante ORAC también, conteniendo unos $835 \mathrm{mg} / 100 \mathrm{~g}^{10}$ de compuestos fenólicos totales (que contribuyen a ORAC-H) y 26,19 mg/100 g de vitamina $\mathrm{E}$ (que contribuye a ORAC-L), siendo este valor muy superior a la de las nueces $(0,8 \mathrm{mg} / 100 \mathrm{~g})^{20}$.

Aunque los datos sobre la ingesta de la capacidad antioxidante de los españoles están incompletos debido a algunas limitaciones, si son orientativos. Si bien una de las debilidades de este trabajo es que hemos empleado para el cálculo teórico de la ingesta media de la capacidad antioxidante de la dieta de los españoles una base de datos americana (USDA). Debido a que la composición de los alimentos es variable de un país a otro y mucho más entre continentes y al amplio conocimiento que se tiene en la actualidad sobre la capacidad antioxidante de los alimentos sobre la salud, se insta a la creación de una base de datos de medida de capacidad antioxidante con metodología ORAC en la que se incluya todos los alimentos utilizados en la cocina de nuestro país (España). Otra limitación es que la ingesta estimada y los valores teóricos de capacidad antioxidante, no tienen en cuenta la biodisponibilidad de los antioxidantes. Por lo que son requeridas más investigaciones que asocien el estado oxidativo en humanos y el riesgo de padecer enfermedades crónicas para poder avanzar en el entendimiento de esta área de investigación y poder establecer unas IDR que promuevan la salud.

\section{CONCLUSIÓN}

La población española supera la recomendación diaria estimada de antioxidantes, siendo incluso superior a otros países que siguen dietas muy similares. El grupo de alimentos que más índice de riqueza de antioxidantes ORAC aporta a la población española es el de las frutas y productos frutícolas, seguido, con poca diferencia, de las verduras y vegetales. A su vez, dentro de estos grupos, son la manzana y la patata los que más contribuyen.

A pesar de ser éstos los grupos que más contribuyen al valor ORAC debido a la cantidad ingerida por persona y día, son los vinos de mesa los que realmente hacen una mayor aportación del índice de riqueza de antioxidantes debido a su composición nutricional

\section{RESUMEN}

En el organismo, las reacciones mediante las cuales se obtienen la energía producen moléculas reactivas de oxígeno (ROS). Los ROS pueden dañar biomoléculas como los lípidos, proteínas, hidratos de carbono, proteínas y el ADN; y esto han sido relacionados con enfermedades crónicas como el cáncer, enfermedades cardiovasculares, procesos inflamatorios, diabetes y el envejecimiento Los antioxidantes presentes en los alimentos pueden contrarrestar el efecto nocivo de los ROS. El objetivo de este estudio fue cuantificar mediante la ingesta media de los españoles de índice de capacidad antioxidante. Para ello se empleó la ingesta media de alimentos por día de los españoles publicada en la Encuesta Nacional de Dietética Española del año 2011 y la base de datos que ha publicado la USDA (basada en el ORAC). La población española superó la recomendación diaria estimada de antioxidantes, siendo incluso superior a otros países que siguen dietas muy similares.

Palabras clave: nutrición; capacidad antioxidante; ORAC; adultos; dieta.

\section{BIBLIOGRAFÍA}

1. Costa ASG, Nunes MA, Almeida IMC, Carvalho MR, Barroso $M F$, Alves RC, Oliveira MBPP. Teas, dietary supplements and fruit juices: a comparative study regarding antioxidant activity 
and bioactive compounds. LWT-Food Sci Technol 2012; 49 (2): 324-328.

2. Tapia A, Rodriguez J, Theoduloz C, López S, Feresin GE, Hirschmann GS. Free radical scavengers and antioxidants from Baccharis grisebachii. J Ethnopharmacol 2004; 95: 155-161.

3. Knasmuller S, Nersesyan A, MisiK M, Gerner C, Mikulits W, Ehrlich V, Wagner KH. Use of convencional an-omics based methods for health claims of dietary antioxidants: Critical reviews. Br J Nutr 2008; 99(E-S1): ES3-ES52.

4. Tachakittirungrod S, Okonogi S, Chowwanapoonpohn S. Study on antioxidant activity of certain plants in Thailand: Mechanism of antioxidant action of guava leave extract. J Food Chem 2007; 103: 381-388.

5. Cooper AJ, Forouhi NG, Ye Z, Buijsee B, Arriola L, Balkau B, Barricarte A, Beulens JWJ et al. Fruit and vegetables intake and type 2 diabetes: EPIC-InterAct prospective study and meta-analysis. Eur J Clin Nutr 2012; 66: 1082-1092.

6. George TW, Paterson E, Waroonphan S, Gordon MH, Lovegrove JA. Effects of chronic consumption of fruit and vegetables puree-based drinks on vasodilation, plasma oxidative stability and antioxidant status. I Hum Nutr Diet 2012; 25: 477-487.

7. Nurul SR, Asmah H. Evaluation of antioxidant properties in fresh and pickled papaya. Int Food Res J 2012; 19: 1117-1124.

8. Scalbert A, William G. Dietary intake and biovailability of polyphenols. I Nutr 2000; 130(8): 2073S-2058S.

9. Prior RL, Cao G. Analysis of botanicals and dietary supplements for antioxidant capacity: A review. J AOAC Int 2000; 83(4): 950-956.

10. Haytowitz DB, Bhagwat S. USDA Database for the Oxygen Radical Absorbance Capacity (ORAC) of Selected Foods, Release 2. United States Department of Agriculture 2010. http://www.orac-info-portal.de/download/ORAC_R2.pdf.

11. Speisky H, López-alarcón C, Gómez M, Fuentes J, SandovalAcuña C. First web-Based Database on total phenolics and oxygen radical absorbance capacity (ORAC) of fruits produced and consumed within the south Andes Region of South America. J Agric Food Chem 2012; 60: 8851-8859.

12. Spanish Food Safety and Nutrition Agency (AESAN). Spanish National Dietary Ingestion Survey 2011. http://docplayer.
es/10558027-Enide-presentacion-n-de-enide-2011-encuestanacional-de-ingesta-dietetica-tica-espanola-aesan-aesan.html.

13. Rothwell JA, Pérez-Jiménez J, Neveu V, Medina-Ramon A, M'Hiri N, Garcia Lobato P, Manach C, Knox K, Eisner R, Wishart D, Scalbert A. Phenol-Explorer 3.0: a major update of the Phenol-Explorer database to incorporate data on the effects of food processing on polyphenol content. Database (Oxford); 2013. https://www.ncbi.n/m.nih.gov/pmc/articles/ PMC 3792339

14. Estévez-Santiago R, Beltrán-de-Miguel B, Olmedilla-Alonso $B$. Assessment of dietary lutein, zeaxanthin and lycopene intakes and sources in the Spanish survey of dietary intake (2009-2010). Int J Food Sci Nutr 2016; 67(3): 305-313.

15. García Alonso FJ, Navarro-González I, Ros G, Periago MJ. Assessment of the antioxidant properties of tomato extracts: a synergistic approach using in vitro chemical tests and cellbased assays. Acta Aliment 2015; 44(2): 297-303.

16. Del Pozo de la Calle S, García Iglesias V, Cuadrado Vives C, Ruiz Moreno E, Valero Gaspar T, Ávila Torres JM, Varela Moreiras G. Nutritional valuation of the spanish diet according to the food consumption panel. Spanish Nutrition Foundation: Lesinguer S.L; 2012.

17. Prior R L, Gu L, Wu X, Jacob RA, Sotoudeh G, Kader A, Cook $R A$. Plasma antioxidant capacity changes following a meal as a measure of the ability of a food to alter in vivo antioxidant status. I Am Coll Nutr 2007; 26: 170-181.

18. Kolomvotsou A, Rallidis SL, Mountzouris K, Lekakis J, Koutelidakis A, Efstathiou S. et al. Adherence to Mediterranean diet and close dietetic supervision increase total dietary antioxidant intake and plasma antioxidant capacity in subjects with abdominal obesity. Eur J Nutr 2013; 52: 37-48.

19. Wu X, Beecher GR, Holden JM, Haytowitz BD, Gebhardt SE, Prior RL. Lipophilic and hydrophilic antioxidant capacities of common foods in the United States. J Agric Food Chem 2004; 52(12): 4026-4037.

20. Spanish Food Composition Database (BEDCA). RedBeDCA y AESAN, Spain; 2006. http://www.bedca.net/bdpub/

21. USDA Food Composition Database. United States Deparment of Agriculture. USA; 2016. https://ndb.nal.usda.gov/ndb 